\title{
openheart Prognosis of patients with secondary mitral regurgitation and reduced ejection fraction
}

\author{
Samer Mowakeaa, ${ }^{1}$ Aeshita Dwivedi, ${ }^{2}$ Jason R Grossman, ${ }^{3}$ Gaurav Parikh, ${ }^{4}$ \\ Zelmira Curillova, ${ }^{5}$ Krishna G Aragam, ${ }^{6,7}$ Sammy Elmariah, ${ }^{6,7}$ Scott Kinlay, ${ }^{6,8,9}$ \\ Jayashri Aragam, ${ }^{6,8}$
}

To cite: Mowakeaa S,

Dwivedi A, Grossman JR, et al. Prognosis of patients with secondary mitral regurgitation and reduced ejection fraction. Open Heart 2018;5:e000745. doi:10.1136/ openhrt-2017-000745

\section{$\mathrm{SM}$ and $\mathrm{AD}$ contributed equally.}

This study was conducted in the Veterans Administration Boston Healthcare System in West Roxbury, Massachusetts, USA. The findings of this study were presented in poster format at the American Society of Echocardiography Scientific Sessions in Minneapolis, Minnesota, USA, 2013.

Received 6 November 2017 Revised 17 November 2017 Accepted 18 November 2017

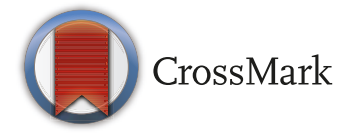

For numbered affiliations see end of article.

\section{Correspondence to}

Dr Jayashri Aragam; Jayashri. Aragam@va.gov

\section{ABSTRACT}

Objective The impact of the severity of secondary mitral regurgitation (MR) on the risk of death and heart failure (HF) hospitalisations in patients with reduced left ventricular (LV) systolic function is poorly defined. The study sought to identify the incremental risk of secondary MR in patients with reduced LV systolic function. Methods We studied 615 consecutive patients with LV ejection fraction $\leq 35 \%$ by transthoracic echocardiography at a single medical centre. Patients were divided into three groups of no MR, mild, or moderate to severe MR. The median follow-up was 2.9 years. The primary endpoint was a composite of death or HF hospitalisations.

Results Compared with patients with no MR, the risk of death or HF hospitalisations was higher for mild MR (HR 1.7, $P=0.003)$ and moderate to severe MR (HR 2.7, $\mathrm{P}<0.001)$. The risk was also higher for the component endpoints of HF hospitalisations (mild MR: HR 2.3, $\mathrm{P}=0.001$; moderate to severe MR: HR 3.5, $\mathrm{P}<0.001$ ) and death (mild MR: HR 1.6, $P=0.033$; moderate to severe MR: HR 2.6, $P<0.001$ ). After adjustment for other covariates, MR was no longer significantly associated with death or HF hospitalisations, or death alone, but remained significantly associated with HF hospitalisations (mild MR: HR 1.7, P=0.028; moderate to severe MR: HR 2.2, $\mathrm{P}=0.002)$.

Conclusions In patients with reduced LV systolic function, secondary MR is associated with an increased risk of HF hospitalisations but not death.

\section{INTRODUCTION}

Patients with reduced left ventricular (LV) systolic function commonly develop malcoaptation of structurally normal leaflets secondary to altered LV geometry, a condition termed secondary mitral regurgitation (MR). Secondary MR is thought to arise from a combination of factors including altered mitral leaflet tethering due to focal or global $\mathrm{LV}$ remodelling, decreased $\mathrm{LV}$ closing forces ${ }^{1}$ and mitral annular dilatation. ${ }^{23}$ The resultant, chronic volume overload imposes additional diastolic wall stress and triggers further LV remodelling and dysfunction thereby perpetuating a vicious cycle. ${ }^{4-6}$ At the cellular and

\section{Key questions}

What is already known about this subject?

- A strong association between the severity of secondary mitral regurgitation (MR) and all-cause mortality as well as hospitalisation for heart failure has been reported. However, the existing data from multiple studies is discordant. It can be debated if secondary MR is independently associated with all-cause mortality beyond that rendered by the underlying cardiomyopathy and other associated comorbidities.

What does this study add?

- Secondary MR is associated with an adverse prognosis in patients with left ventricular systolic dysfunction. However, its impact independent of the underlying cardiomyopathy and other covariates may be on overall morbidity but not on mortality.

How might this impact on clinical practice?

- Based on these findings, we believe that interventions to reduce secondary MR in this patient population may improve the qualitybut not the quantity—of life. This has clinical implications in the selection of patients undergoing therapies targeted towards mitral valve repair or replacement.

molecular levels, this pathological process is mediated by excess neurohormonal and cytokine activation, loss of cardiomyocytes and inadequate hypertrophic compensation of the left ventricle.

Despite these deleterious effects, it remains unclear if secondary MR confers an incremental risk beyond that rendered by the underlying cardiomyopathy. While some studies have identified secondary MR as an independent risk factor for increased mortality, ${ }^{7-11}$ others suggest that the underlying cardiomyopathy primarily drives mortality risk. ${ }^{12-15}$ The controversy may impact the decision to pursue aggressive 
management of secondary MR via surgical or transcatheter repair or replacement of the mitral valve. ${ }^{16}$

The primary objective of our study was to examine the incremental prognostic impact of secondary MR on all-cause mortality and hospitalisations for heart failure (HF) in patients with reduced LV systolic function.

\section{METHODS}

\section{Patient selection}

We reviewed the medical records of patients from the Veterans Affairs (VA) Boston Healthcare System database. Using the Crystal Reports analysis software programme, we included consecutive patients with a left ventricular ejection fraction (LVEF) $\leq 35 \%$ who received a transthoracic echocardiographic (TTE) study for clinically indicated purposes between 1 April 2009 and 31 December 2011. We excluded patients with (1) structural mitral valve disease (more than mild mitral stenosis or prolapse as well as rheumatic or degenerative mitral valve regurgitation), (2) more than mild aortic stenosis or regurgitation and (3) history of mitral valve repair or replacement.

Patient demographics, comorbid conditions and clinical outcomes data-including all-cause mortality and hospitalisations for $\mathrm{HF}$-were collected through retrospective chart review. All charts were reviewed for outcomes data through February 2013. Blood pressure, body surface area, inpatient versus outpatient status and echocardiographic variables were collected from the TTE reports at the time of the index study. Renal function was assessed by the glomerular filtration rate estimated from the Cockroft-Gault equation. Causes of reduced LV systolic function were determined from the medical chart and categorised as ischaemic, non-ischaemic, mixed (ischaemic and non-ischaemic) and unknown. TTE studies were interpreted by board-certified echocardiographers with substantial clinical experience in echocardiography. Given the retrospective nature of the study, no informed consent was required.

\section{Grading MR severity}

As any single parameter should not be relied on while grading severity of MR, ${ }^{17}$ a systematic analysis of the MR severity was performed integrating the following elements as recommended by American Society of Echocardiography ${ }^{17}$ : Vena contracta width (mild when $<0.3 \mathrm{~cm}$, moderate when $0.3-0.6 \mathrm{~cm}$ and severe when $\geq 0.7 \mathrm{~cm}$ ), effective regurgitant orifice area (mild when $<20 \mathrm{~mm}^{2}$, moderate when $20-39 \mathrm{~mm}^{2}$ and severe when $\geq 40 \mathrm{~mm}^{2}$ ) and regurgitant volume (mild when $<30 \mathrm{~mL}$, moderate when $30-59 \mathrm{~mL}$ and severe when $\geq 60 \mathrm{~mL}$ ), pulmonary venous flow pattern, mitral inflow velocity, density of the MR continuous wave Doppler profile, as well as the MR jet area. Although these parameters were performed in all patients, except for a few with poor image quality, their respective values were not solely relied on in the final determination of MR severity, but rather integrated together for the final interpretation. Evaluation of left atrial (LA) size was used as additional data for grading MR severity. ${ }^{17}$ LA size was determined by volume assessments as calculated by the biplane area-length formula. ${ }^{18} \mathrm{LA}$ size was graded based on the LA volume indexed to body surface area (normal when LA volume index $<29 \mathrm{~mL}$ / $\mathrm{m}^{2}$, mildly dilated when LA volume index $29-33 \mathrm{~mL} / \mathrm{m}^{2}$, moderately dilated when LA volume index $34-39 \mathrm{~mL}$ / $\mathrm{m}^{2}$ and severely dilated when LA volume index $\geq 40 \mathrm{~mL} /$ $\left.\mathrm{m}^{2}\right) .^{18}$

Based on this grading method, patients enrolled in the study were further categorised into three main groups according to MR severity (no MR, mild MR, and moderate to severe MR). For purposes of analysis in our study, patients with moderate MR were combined with patients with severe MR in one group to increase the sample size.

Severity of tricuspid valve regurgitation was assessed by using colour flow and spectral Doppler imaging according to the guidelines proposed by the American Society of Echocardiography. ${ }^{17}$

\section{Endpoints}

The primary endpoint was a composite of all-cause death and hospitalisations for HF. A hospitalisation for HF was defined as any hospital admission during which the patient was identified to have symptoms of acute HF exacerbation (eg, breathlessness, fatigue or ankle swelling) with consistent physical exam findings as documented in the chart (eg, rales on lung exam, elevated jugular venous pressure or peripheral oedema) that required intravenous diuretic therapy. Secondary endpoints included the individual component outcomes of all-cause death and hospitalisation for HF.

\section{Statistical analysis}

Continuous variables were expressed as mean and SD or median and IQR. Associations between MR category and continuous and categorical variables were studied using analysis of variance and $\mathrm{X}^{2}$ tests, respectively. Survival curves for death or hospitalisation for HF were assessed by the Kaplan-Meier method and the differences between event rates were tested for significance using the log-rank test. The time to primary and secondary endpoints was analysed using univariable and multivariable Cox proportional hazards models. Patients were censored at their last documented encounter in the electronic record. Clinically relevant variables were included in a backward stepwise multivariate model $(\mathrm{P}$ for removal $>0.05)$ to determine the variables independently associated with the primary endpoint. A $\mathrm{P}$ value $<0.05$ was considered statistically significant.

\section{RESULTS}

\section{Baseline characteristics}

A total of 815 patients were reviewed, of which 200 patients did not meet inclusion criteria and the remaining 615 patients (mean age $69 \pm 11$ ) were analysed. The median follow-up duration for the study was 2.9 years (IQR $0.8-3.7$ years). There were 179 patients (29\%) with no 
Table 1 Baseline characteristics by severity of mitral regurgitation (MR)

\begin{tabular}{|c|c|c|c|c|}
\hline & $\begin{array}{l}\text { No } \\
\text { MR }(n=179)\end{array}$ & $\begin{array}{l}\text { Mild } \\
\text { MR }(n=192)\end{array}$ & $\begin{array}{l}\text { Moderate } \\
\text { to severe } \\
\text { MR }(n=244)\end{array}$ & $P$ value \\
\hline Age (years), mean (SD) & $66(10)$ & $70(10)$ & $71(11)$ & $<0.0001$ \\
\hline Male, n (\%) & $177(99)$ & $184(96)$ & $240(98)$ & 0.1 \\
\hline Body surface area $\left(\mathrm{m}^{2}\right)$, mean (SD) & $2.1(0.3)$ & $2.0(0.2)$ & $2.0(0.2)$ & 0.0001 \\
\hline Systolic blood pressure (mm Hg), mean (SD) & $123(21)$ & $123(20)$ & $119(20)$ & 0.076 \\
\hline Diastolic blood pressure (mm Hg), mean (SD) & $71(12)$ & $73(15)$ & $70(14)$ & 0.16 \\
\hline Hypertension, n (\%) & $142(79)$ & $161(84)$ & $195(80)$ & 0.489 \\
\hline Diabetes mellitus, $\mathrm{n}(\%)$ & $73(41)$ & $95(49)$ & $93(38)$ & 0.051 \\
\hline Atrial fibrillation, $\mathrm{n}(\%)$ & $52(29)$ & $65(34)$ & $100(41)$ & 0.035 \\
\hline History of CAD, $\mathrm{n}(\%)$ & $112(63)$ & $139(72)$ & $175(72)$ & 0.068 \\
\hline History of CABG surgery, $\mathrm{n}(\%)$ & $42(24)$ & $73(38)$ & $78(32)$ & 0.041 \\
\hline Diuretic use, $\mathrm{n}(\%)$ & $89(50)$ & $110(57)$ & $167(68)$ & $<0.001$ \\
\hline ACE inhibitor use, $\mathrm{n}(\%)$ & $123(69)$ & $142(74)$ & $119(49)$ & $<0.001$ \\
\hline ARB use, $n(\%)$ & $17(9.6)$ & $18(9.4)$ & $39(16)$ & 0.072 \\
\hline Beta-blocker use, n (\%) & $155(88)$ & $158(82)$ & $200(82)$ & 0.235 \\
\hline Glomerular filtration rate $\left(\mathrm{mL} / \mathrm{min} / 1.73 \mathrm{~m}^{2}\right)$, mean (SD) & $94(44)$ & $78(39)$ & $67(34)$ & $<0.0001$ \\
\hline History of implantable cardioverter defibrillator, n (\%) & $38(21)$ & $42(22)$ & $54(22)$ & 0.975 \\
\hline History of biventricular pacemaker implant, $\mathrm{n}(\%)$ & $20(11)$ & $21(11)$ & $22(9)$ & 0.716 \\
\hline Statin use, $\mathrm{n}(\%)$ & $139(78)$ & $157(82)$ & $164(67)$ & 0.002 \\
\hline \multicolumn{5}{|l|}{ Cardiomyopathy } \\
\hline Ischaemic cardiomyopathy, n (\%) & $111(62)$ & $130(68)$ & $157(64)$ & \multirow[t]{4}{*}{0.729} \\
\hline Non-ischaemic cardiomyopathy, n (\%) & $52(29)$ & $48(25)$ & $59(24)$ & \\
\hline Mixed cardiomyopathy, n (\%) & $8(5)$ & $6(3)$ & $12(5)$ & \\
\hline Unknown cause of cardiomyopathy, $\mathrm{n}(\%)$ & $8(4)$ & $8(4)$ & $16(7)$ & \\
\hline LVIDd (cm), mean (SD) & $5.7(0.7)$ & $5.7(0.8)$ & $5.9(0.8)$ & 0.0007 \\
\hline LVIDs (cm), mean (SD) & $4.7(0.8)$ & $4.8(0.8)$ & $5.1(0.9)$ & $<0.0001$ \\
\hline Left atrial dimension (cm), mean (SD) & $4.1(0.6)$ & $4.2(0.6)$ & $4.5(0.6)$ & $<0.0001$ \\
\hline \multicolumn{5}{|l|}{ Left atrial volume indexed to body surface area $\left(\mathrm{mL} / \mathrm{m}^{2}\right)$} \\
\hline Normal left atrial size $\left(\mathrm{LAVI}<29 \mathrm{~mL} / \mathrm{m}^{2}\right), \mathrm{n}(\%)$ & $67(38)$ & $44(23)$ & $20(8)$ & \multirow[t]{4}{*}{$<0.0001$} \\
\hline Mildly dilated LA (LAVI $\left.29-33 \mathrm{~mL} / \mathrm{m}^{2}\right), \mathrm{n}(\%)$ & $59(33)$ & $49(26)$ & $56(23)$ & \\
\hline Moderately dilated (LAVI 34-39 mL/m²), n (\%) & $30(17)$ & $54(28)$ & $75(31)$ & \\
\hline Severely dilated $\left(\mathrm{LAVl} \geq 40 \mathrm{~mL} / \mathrm{m}^{2}\right), \mathrm{n}(\%)$ & $22(12)$ & $44(23)$ & $92(38)$ & \\
\hline LVEF $(\%)$, median $(25 \%, 75 \%)$ & $30(26,33)$ & $28(23,33)$ & $25(20,33)$ & $<0.0001$ \\
\hline Pulmonary artery systolic pressure (mm Hg), mean (SD) & $28(9)$ & $31(12)$ & $37(12)$ & $<0.0001$ \\
\hline Pulmonary venous systolic flow reversal, $n(\%)$ & $18(11)$ & $43(23)$ & $60(25)$ & $<0.001$ \\
\hline \multicolumn{5}{|l|}{ Tricuspid regurgitation } \\
\hline None, $\mathrm{n}(\%)$ & $130(73)$ & $98(51)$ & $53(22)$ & \multirow[t]{4}{*}{$<0.001$} \\
\hline Mild, n (\%) & $33(18)$ & $62(32)$ & $75(31)$ & \\
\hline Moderate, n (\%) & $11(6)$ & $23(12)$ & $80(33)$ & \\
\hline Severe, n (\%) & $5(3)$ & $8(4)$ & $35(14)$ & \\
\hline
\end{tabular}

ARB, angiotensin receptor blocker; CAD, coronary artery disease; CABG, coronary artery bypass graft; LA, left atrial; LAVI, LA volume indexed to body surface area (LA volume could not be measured in one person in each group); LVEF, left ventricular ejection fraction; LVIDd, left ventricular internal dimension at end diastole; LVIDs, left ventricular internal dimension at end systole. 

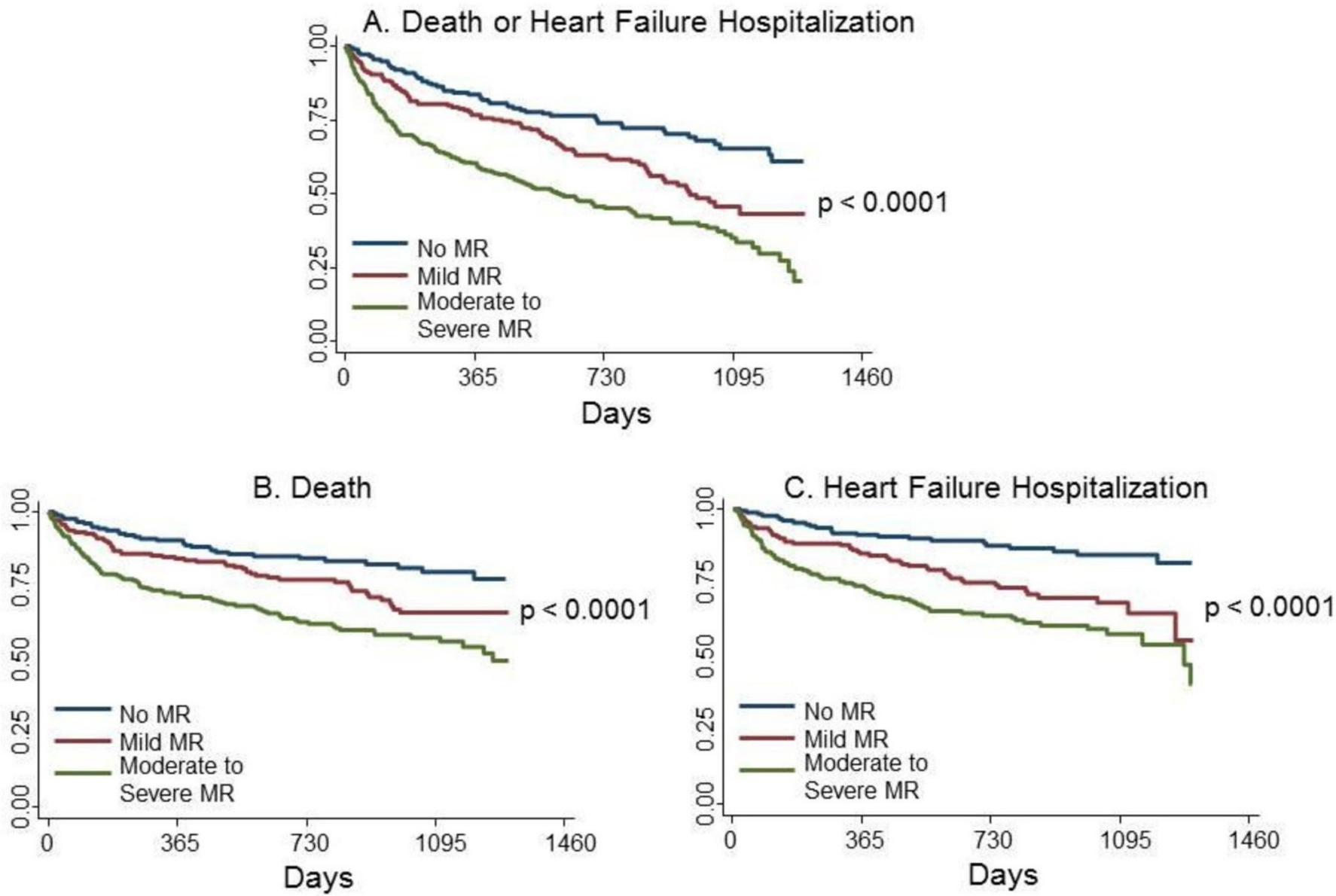

Figure 1 Survival curves by severity of mitral regurgitation (MR) for (A) death or heart failure hospitalisation, (B) death and (C) heart failure hospitalisation.

MR, 192 patients $(31 \%)$ with mild MR and 244 patients $(40 \%)$ with moderate to severe MR.

Clinical and echocardiographic characteristics of patients in the different groups are shown in table 1. As patients were enrolled from a VA medical centre, most patients were male $(97.7 \%)$. Patients with increasing severity of MR were more likely to be older $(\mathrm{P}<0.0001)$, have a history of atrial fibrillation $(\mathrm{P}=0.035)$ and coronary artery bypass graft $(\mathrm{CABG})$ surgery $(\mathrm{P}=0.04)$ as well as a significantly lower glomerular filtration rate $(\mathrm{P}<0.0001)$. MR severity was not associated with the underlying cause of cardiomyopathy (ischaemic vs non-ischaemic) $(\mathrm{P}=0.73)$. Severity of MR directly correlated with left ventricle internal dimensions at end diastole (LVIDd) and end systole (LVIDs), with LA dimensions and indexed LA volumes, but was inversely related to the LVEF $(\mathrm{P}<0.0001)$. Additionally, patients with more severe MR had significantly higher pulmonary artery systolic pressures $(\mathrm{P}<0.0001)$ and more severe tricuspid regurgitation $(\mathrm{P}=0.001)$, consistent with the results of prior studies (table 1). ${ }^{9}$

\section{Outcomes}

A total of 287 patients (47\%) reached the primary composite endpoint of death or HF hospitalisation during the follow-up period. Death occurred in 184 patients $(30 \%)$, while a hospitalisation for HF occurred in 160 patients (26\%). Survival curves for the combined and component endpoints are shown in figure 1, and outcomes were worse with more severe MR.

\section{Survival analysis}

In univariable analyses, death or HF hospitalisation was significantly related to mild MR (HR 1.7; 95\% CI 1.2 to 2.4, $\mathrm{P}=0.003$ ) and moderate to severe MR (HR 2.7; 95\% CI 2.0 to $3.7, \mathrm{P}<0.001$ ) (table 2 ). MR severity was also associated with the component endpoints of hospitalisation for HF (table 3) and death alone (table 4).

Factors significantly associated with the composite primary endpoint included age, inpatient status, any degree of tricuspid regurgitation, history of diabetes mellitus, ACE inhibitor use, diuretic use, atrial fibrillation, LVEF, LVIDd and glomerular filtration rate. After controlling for these variables in a multivariable analysis, the relationship between MR and death or HF hospitalisation was no longer significant (table 2). Similarly, after adjustment of the covariates, there was no significant relationship between MR and death alone (table 4), but MR remained associated with hospitalisations for HF (table 3).

\section{DISCUSSION}

Patients with reduced LV systolic function often develop secondary MR due to associated ventricular 
Table 2 Severity of mitral regurgitation and the risk of death or heart failure hospitalisation

\begin{tabular}{|c|c|c|c|c|c|c|}
\hline \multirow[b]{2}{*}{ Mitral regurgitation } & \multicolumn{3}{|c|}{ Univariable } & \multicolumn{3}{|c|}{ Multivariable } \\
\hline & HR & $95 \% \mathrm{Cl}$ & P value & HR & $95 \% \mathrm{Cl}$ & $P$ value \\
\hline None & 1.0 (ref) & & & 1.0 (ref) & & \\
\hline Mild & 1.7 & 1.2 to 2.4 & 0.003 & 1.2 & 0.9 to 2.5 & 0.24 \\
\hline Moderate to severe & 2.7 & 2.0 to 3.7 & $<0.001$ & 1.3 & 0.9 to 1.9 & 0.11 \\
\hline
\end{tabular}

Multivariable model adjusted for age, tricuspid regurgitation, diabetes mellitus, ACE inhibitors, diuretics, left ventricular ejection fraction and glomerular filtration rate.

and atrial remodelling, with ultimate progression of this cyclical, pathological process to worsening clinical heart failure and death. ${ }^{15} 19-22$ Whether the presence of MR in this population contributes incremental risk beyond that conferred by the underlying cardiomyopathy is unclear. In our study of consecutive patients with reduced systolic function (LVEF $\leq 35 \%$ ), the presence of secondary MR was associated with death and HF hospitalisation, but the relationship to this combined endpoint and to death alone was attenuated by adjustment for other covariates. However, the severity of MR was related to HF hospitalisation before and after adjustment for covariates. This suggests a more direct role for the severity of MR on HF hospitalisation, but that the risk of death is related to covariates that associate with MR. Thus, trials of surgical or catheter-based treatments of MR are more likely to reduce HF hospitalisation, but less likely to affect death.

Prior studies have produced conflicting data on the independent impact of secondary MR on morbidity and mortality in patients with $\mathrm{LV}$ systolic dysfunction. ${ }^{7-15}$ Fundamental differences in the cohorts and methodologies employed have likely resulted in divergent study conclusions and continued clinical uncertainty.

Among 558 consecutive patients with advanced $\mathrm{LV}$ systolic dysfunction and HF due to ischaemic and non-ischaemic cardiomyopathy, Patel and colleagues found that secondary MR was not independently associated with mortality, although the authors did not examine the risk of recurrent hospitalisations. ${ }^{14}$ In a prospective multicenter study of 336 patients with New York Heart Association functional class III-IV HF and LVEF of $\leq 35 \%$, the severity of secondary MR did not predict death, but independently predicted the composite endpoint of death, HF hospitalisations or transplant. ${ }^{13}$
In contrast, in a more recent study of 1256 patients with ischaemic and non-ischaemic cardiomyopathy and severe LV dysfunction, quantitatively determined secondary MR was an independent predictor of death or HF hospitalisation at a median follow-up of 2.5 years. ${ }^{10}$ Multivariable analysis in this study did not include clinical factors such as diabetes mellitus, renal function or medication use-covariates that, when employed in our multivariable model, rendered the association between MR and death or HF hospitalisation no longer statistically significant.

A separate study of 303 patients with previous myocardial infarction ( $>16$ days) followed for 5 years demonstrated an association between secondary MR and excess mortality. ${ }^{8}$ In a subsequent analysis of 173 patients with prior myocardial infarction ( $>16$ days), secondary MR was associated with a threefold increase in risk of congestive heart failure and a higher incidence of the combined endpoint of death or HF after approximately 4 years of follow-up. ${ }^{23}$

Several reasons have been posited for the variability in these study results but most relate to the complex interplay between secondary MR, LV dysfunction and remodelling. In particular, while possible to adjust for LVEF, it is difficult to control for variations in ventricular remodelling, which may contribute more to longterm risk than associated secondary MR. In fact, of the above studies that reported excess mortality with secondary MR, most were composed of patients with a greater burden of myocardial ischaemia and/or prior infarction-a distinct clinical profile that may be predisposed to poorer outcomes.

Despite discrepant data on clinical outcomes, the consensus remains that secondary MR is associated with an adverse prognosis and therefore warrants targeted therapy. ${ }^{24}$ While a causal link between secondary MR and

Table 3 Severity of mitral regurgitation and the risk of heart failure hospitalisation alone

\begin{tabular}{|c|c|c|c|c|c|c|}
\hline \multirow[b]{2}{*}{ Mitral regurgitation } & \multicolumn{3}{|c|}{ Univariable } & \multicolumn{3}{|c|}{ Multivariable } \\
\hline & HR & $95 \% \mathrm{Cl}$ & $P$ value & HR & $95 \% \mathrm{CI}$ & $P$ value \\
\hline None & 1.0 (ref) & & & 1.0 (ref) & & \\
\hline Moderate to severe & 3.5 & 2.2 to 5.5 & $<0.001$ & 2.2 & 1.3 to 3.6 & 0.002 \\
\hline
\end{tabular}

Multivariable model adjusted for tricuspid regurgitation, diabetes mellitus, ACE inhibitors, diuretics, atrial fibrillation and glomerular filtration rate. 
Table 4 Severity of mitral regurgitation and the risk of death alone

\begin{tabular}{|c|c|c|c|c|c|c|}
\hline \multirow[b]{2}{*}{ Mitral regurgitation } & \multicolumn{3}{|c|}{ Univariable } & \multicolumn{3}{|c|}{ Multivariable } \\
\hline & HR & $95 \% \mathrm{Cl}$ & $P$ value & HR & $95 \% \mathrm{Cl}$ & $P$ value \\
\hline None & 1.0 (ref) & & & 1.0 (ref) & & \\
\hline Mild & 1.6 & 1.0 to 2.5 & 0.033 & 1.1 & 0.7 to 1.7 & 0.70 \\
\hline Moderate to severe & 2.6 & 1.7 to 3.8 & $<0.001$ & 1.3 & 0.8 to 2.0 & 0.28 \\
\hline
\end{tabular}

Multivariable model adjusted for age, tricuspid regurgitation, diabetes mellitus, ACE inhibitors, left ventricular ejection fraction and glomerular filtration rate.

mortality would imply that repair of the mitral valve might improve long-term survival, this hypothesis is relatively unsupported by contemporary surgical literature. ${ }^{25-32}$ In a retrospective study of 390 propensity-matched patients with 3-4+ secondaryMR and LVEF $<45 \%$ undergoing CABG, concurrent mitral valve repair was associated with reduced postoperative MR and improved symptoms, but not with improved long-term functional status or survival. ${ }^{27}$ Similarly, in a study of 107 patients with severe $\mathrm{LV}$ dysfunction (mean LVEF $35 \% \pm 9 \%$ ) and moderate to severe secondary MR, mitral valve repair in addition to CABG was effective in eliminating MR, but did not differ from CABG alone in 5 year actuarial survival rates $(88 \mathrm{vs}$ $87 \%){ }^{26}$

More recently, a large retrospective study of 4989 patients with significant $\mathrm{CAD}$, moderate $\mathrm{LV}$ dysfunction (mean $\mathrm{LVEF}=40 \%-50 \%$ ) and moderate to severe secondary MR assessed the relative survival advantages associated with medical therapy $(\mathrm{n}=1800)$, percutaneous coronary intervention $(n=1295)$, CABG alone $(n=1651)$ and $\mathrm{CABG}$ with mitral valve repair or replacement $(\mathrm{n}=243)$ over a 20-year period. At a median follow-up of 5.3 years, isolated CABG was associated with the highest adjusted survival (adjusted HR 0.56 ; 95\% CI 0.51 to 0.62 , $\mathrm{P}<0.0001)$ when compared with medical therapy, and no additional survival benefit was conferred by concomitant mitral valve repair or replacement irrespective of secondary MR severity. The study highlights that longterm survival may be more dependent on relief of ischaemia than on correction of secondary MR, suggesting that both MR and its associated mortality may be driven by pathological remodelling-particularly in the setting of ischaemic heart disease-and a poor ventricular substrate. $^{29}$

Notably, in the Surgical Treatment for Ischaemic Heart Failure trial assessing medical therapy versus CABG among patients with ischaemic cardiomyopathy and $\mathrm{LVEF} \leq 35 \%$, the addition of mitral valve repair to CABG $(n=49)$ in patients with moderate to severe MR was associated with improved survival. However, apart from the small sample size and a low median LVEF (25\%), mitral valve repair in this study was pursued in a non-randomised fashion at the surgeon's discretion inducing a possible selection bias wherein surgery was avoided for the relatively sicker study patients. ${ }^{33}$

The largest and most recent prospective randomised trial included 301 patients with moderate ischaemic
MR randomly assigned at 26 sites to $\mathrm{CABG}$ alone $(n=151)$ or CABG and mitral valve repair $(n=150)$. The primary endpoint assessed was LV remodelling as measured by end systolic volume index at the end of 1 year and secondary endpoints included a composite of major adverse cardiac or cerebrovascular events and individual endpoints such as mortality, functional status and quality of life. The study did not reveal a difference in LV remodelling. MR was decreased in the group that underwent mitral valve repair and CABG but there was no significant difference between the two groups in major adverse cardiac or cerebrovascular events or in survival at 12 months. ${ }^{32}$ Two earlier randomised trials of $\mathrm{CABG} \pm$ mitralvalve repair demonstrated reduced secondary MR and improved symptoms with mitral valve repair but were underpowered to determine an effect on mortality. ${ }^{30} 31$

\section{Clinical implications and future directions}

Our study supports the hypothesis that secondary MR in the setting of LV dysfunction influences morbidity reflected by HF hospitalisation, but not mortality. Current guidelines recommend aggressive medical therapy as the cornerstone of secondary MR management in patients with severe cardiomyopathy, followed by CRT and revascularisation in those who meet clinical indications. ${ }^{34}$ Mitral valve repair or replacement may play a role in patients who are refractory to the above therapies. As the goals of therapy often shift in patients with advanced heart failure, mitral valve repair or replacement in the appropriate context may promote improved quality over quantity of life. The ongoing Cardiovascular Outcomes Assessment of the MitraClip Percutaneous Therapy for Heart Failure Patients with Functional Mitral Regurgitation trial assessing a catheter-based mitral valve therapy in patients with HF with significant secondary MR will help determine the efficacy of mitral valve repair. Our study would suggest that it will have a greater effect on HF hospitalisation than on death.

\section{Strengths and limitations}

The major strength of this study is the inclusion of a broad list of patient comorbidities as well as relevant medications and echocardiographic variables which were included in the multivariable analysis. In addition, a comprehensive integrative approach in the assessment of MR severity was carried out in all study patients, as 
recommended by most recent American Society of Echocardiography guidelines. ${ }^{17}$

Several limitations, however, must be acknowledged. Our study was retrospective and $97.8 \%$ of the patients in the study were men reflecting a typical VA population. In addition, complete coronary angiography data was not available in all patients; thus, we were unable to account for the extent of coronary artery disease in our analysis. Finally, specific causes of death, particularly from cardiovascular causes, were not accounted for in our analysis.

\section{CONCLUSION}

In a large, consecutive population of veterans with reduced LV systolic function, the severity of secondary MR was associated death or HF hospitalisation. After risk adjustment by other covariates, the relationship of MR to death was no longer significant. However, MR remained significantly associated with the risk of HF hospitalisation in multivariable models. Thus, interventions to reduce secondary MR in patients with reduced LV systolic function may have a greater effect on HF hospitalisation than death.

\section{Author affiliations \\ ${ }^{1}$ Cardiology, Loyola University Medical Center, Maywood, Illinois, USA \\ ${ }^{2}$ Cardiology, New York University Langone Medical Center, New York, New York, USA ${ }^{3}$ Cardiology, Lahey Hospital and Medical Center, Burlington, Massachusetts, USA ${ }^{4}$ Cardiology, Pomona Valley Hospital Medical Center, Pomona, California, USA ${ }^{5}$ Cardiology, Eastern Maine Medical Center, Bangor, Maine, USA \\ ${ }^{6}$ Cardiology, Harvard Medical School, Boston, Massachusetts, USA ${ }^{7}$ Cardiology, Massachusetts General Hospital, Boston, Massachusetts, USA ${ }^{8}$ Cardiology, VA Boston Healthcare System, West Roxbury, Massachusetts, USA \\ ${ }^{9}$ Cardiology, Brigham and Women's Hospital, Boston, Massachusetts, USA}

Contributors SM and AD: study design and interpretation of data; drafting of manuscript. JRG, KGA and SE: interpretation of data and revision of manuscript. GP: study design and revision of manuscript. ZC: study design and interpretation of data; revision of manuscript. SK: analysis and interpretation of data; revision of manuscript. JA: study design and analysis/interpretation of data; revision of manuscript.

Competing interests None declared.

Ethics approval The VA Central Institutional Review Board.

Provenance and peer review Not commissioned; internally peer reviewed.

Data sharing statement No additional data are available.

Open Access This is an Open Access article distributed in accordance with the Creative Commons Attribution Non Commercial (CC BY-NC 4.0) license, which permits others to distribute, remix, adapt, build upon this work non-commercially, and license their derivative works on different terms, provided the original work is properly cited and the use is non-commercial. See: http://creativecommons.org/ licenses/by-nc/4.0/

(c) Article author(s) (or their employer(s) unless otherwise stated in the text of the article) 2018. All rights reserved. No commercial use is permitted unless otherwise expressly granted.

\section{REFERENCES}

1. Ray S. The echocardiographic assessment of functional mitral regurgitation. Eur J Echocardiogr 2010;11:111-7.

2. Boltwood CM, Tei C, Wong M, et al. Quantitative echocardiography of the mitral complex in dilated cardiomyopathy: the mechanism of functional mitral regurgitation. Circulation 1983;68:498-508.
3. Popović ZB, Martin M, Fukamachi K, et al. Mitral annulus size links ventricular dilatation to functional mitral regurgitation. J Am Soc Echocardiogr 2005;18:959-63.

4. Spinale FG, Ishihra K, Zile M, et al. Structural basis for changes in left ventricular function and geometry because of chronic mitral regurgitation and after correction of volume overload. J Thorac Cardiovasc Surg 1993;106:1147-57.

5. Tsutsui H, Spinale FG, Nagatsu M, et al. Effects of chronic beta-adrenergic blockade on the left ventricular and cardiocyte abnormalities of chronic canine mitral regurgitation. J Clin Invest 1994;93:2639-48.

6. Urabe $\mathrm{Y}, \mathrm{Mann} \mathrm{DL}$, Kent RL, et al. Cellular and ventricular contractile dysfunction in experimental canine mitral regurgitation. Circ Res 1992;70:131-47.

7. Cioffi G, Tarantini L, De Feo S, et al. Functional mitral regurgitation predicts 1-year mortality in elderly patients with systolic chronic heart failure. Eur J Heart Fail 2005;7:1112-7.

8. Grigioni F, Enriquez-Sarano M, Zehr KJ, et al. Ischemic mitral regurgitation: long-term outcome and prognostic implications with quantitative Doppler assessment. Circulation 2001;103:1759-64.

9. Koelling TM, Aaronson KD, Cody RJ, et al. Prognostic significance of mitral regurgitation and tricuspid regurgitation in patients with left ventricular systolic dysfunction. Am Heart J 2002;144:524-9.

10. Rossi A, Dini FL, Faggiano P, et al. Independent prognostic value of functional mitral regurgitation in patients with heart failure. A quantitative analysis of 1256 patients with ischaemic and nonischaemic dilated cardiomyopathy. Heart 2011;97:1675-80.

11. Trichon BH, Felker GM, Shaw LK, et al. Relation of frequency and severity of mitral regurgitation to survival among patients with left ventricular systolic dysfunction and heart failure. Am J Cardiol 2003;91:538-43.

12. Giannuzzi P, Temporelli PL, Bosimini E, et al. Independent and incremental prognostic value of Doppler-derived mitral deceleration time of early filling in both symptomatic and asymptomatic patients with left ventricular dysfunction. J Am Coll Cardiol 1996;28:383-90.

13. Grayburn PA, Appleton CP, DeMaria AN, et al. Echocardiographic predictors of morbidity and mortality in patients with advanced heart failure: the Beta-blocker Evaluation of Survival Trial (BEST). J Am Coll Cardiol 2005;45:1064-71.

14. Patel JB, Borgeson DD, Barnes ME, et al. Mitral regurgitation in patients with advanced systolic heart failure. J Card Fail 2004;10:285-91.

15. Rihal CS, Nishimura RA, Hatle LK, et al. Systolic and diastolic dysfunction in patients with clinical diagnosis of dilated cardiomyopathy. Relation to symptoms and prognosis. Circulation 1994;90:2772-9.

16. Carabello BA. The current therapy for mitral regurgitation. J Am Coll Cardiol 2008;52:319-26.

17. Zoghbi WA, Adams D, Bonow RO, et al. Recommendations for noninvasive evaluation of native valvular regurgitation: a report from the American Society of Echocardiography developed in collaboration with the Society for Cardiovascular Magnetic Resonance. J Am Soc Echocardiogr 2017;30:303-71.

18. Lang RM, Bierig M, Devereux RB, et al. Recommendations for chamber quantification: a report from the American Society of Echocardiography's Guidelines and Standards Committee and the Chamber Quantification Writing Group, developed in conjunction with the European Association of Echocardiography, a branch of the European Society of Cardiology. J Am Soc Echocardiogr 2005;18:1440-63.

19. Factor SM, Sonnenblick EH. The pathogenesis of clinical and experimental congestive cardiomyopathies: recent concepts. Prog Cardiovasc Dis 1985;27:395-420.

20. Francis GS, Goldsmith SR, Levine TB, et al. The neurohumoral axis in congestive heart failure. Ann Intern Med 1984;101:370-6.

21. Zelis R, Flaim SF, Liedtke AJ, et al. Cardiocirculatory dynamics in the normal and failing heart. Annu Rev Physiol 1981;43:455-76.

22. Tikkanen I, Fyhrquist $F$, Metsärinne $\mathrm{K}$, et al. Plasma atrial natriuretic peptide in cardiac disease and during infusion in healthy volunteers. Lancet 1985;2:66-9.

23. Grigioni F, Detaint $\mathrm{D}$, Avierinos JF, et al. Contribution of ischemic mitral regurgitation to congestive heart failure after myocardial infarction. J Am Coll Cardiol 2005;45:260-7.

24. Nishimura RA, Otto CM, Bonow RO, et al. 2014 AHA/ACC guideline for the management of patients with valvular heart disease: a report of the American College of Cardiology/American Heart Association Task Force on Practice Guidelines. J Am Coll Cardiol 2014;63:e57-185.

25. Wu AH, Aaronson KD, Bolling SF, et al. Impact of mitral valve annuloplasty on mortality risk in patients with mitral regurgitation 
and left ventricular systolic dysfunction. J Am Coll Cardiol 2005;45:381-7.

26. Kang DH, Kim MJ, Kang SJ, et al. Mitral valve repair versus revascularization alone in the treatment of ischemic mitral regurgitation. Circulation 2006;114:I-499-0.

27. Mihaljevic T, Lam BK, Rajeswaran J, et al. Impact of mitral valve annuloplasty combined with revascularization in patients with functional ischemic mitral regurgitation. J Am Coll Cardiol 2007;49:2191-201.

28. Schurr P, Boeken $\mathrm{U}$, Limathe J, et al. Impact of mitral valve repair in patients with mitral regurgitation undergoing coronary artery bypass grafting. Acta Cardiol 2010;65:441-7.

29. Castleberry AW, Williams JB, Daneshmand MA, et al. Surgical revascularization is associated with maximal survival in patients with ischemic mitral regurgitation: a 20 -year experience. Circulation 2014;129:2547-56.

30. Fattouch K, Guccione F, Sampognaro R, et al. POINT: efficacy of adding mitral valve restrictive annuloplasty to coronary artery bypass grafting in patients with moderate ischemic mitral valve regurgitation: a randomized trial. $J$ Thorac Cardiovasc Surg 2009;138:278-85.

31. Chan KM, Punjabi PP, Flather M, et al. Coronary artery bypass surgery with or without mitral valve annuloplasty in moderate functional ischemic mitral regurgitation: final results of the Randomized Ischemic Mitral Evaluation (RIME) trial. Circulation 2012;126:2502-10.

32. Smith PK, Puskas JD, Ascheim DD, et al. Surgical treatment of moderate ischemic mitral regurgitation. $N$ Engl $J$ Med 2014;371:2178-88.

33. Deja MA, Grayburn PA, Sun B, et al. Influence of mitral regurgitation repair on survival in the surgical treatment for ischemic heart failure trial. Circulation 2012;125:2639-48.

34. Asgar AW, Mack MJ, Stone GW. Secondary mitral regurgitation in heart failure: pathophysiology, prognosis, and therapeutic considerations. J Am Coll Cardiol 2015;65:1231-48. 